\title{
3 Wiener Judentum und Wiener Sport in der Zwischenkriegszeit: Fakten und Zahlen
}

\section{Die jüdische Bevölkerung Wiens in der Zwischen- kriegszeit: ein demografischer Überblick}

\section{0 bis 1923}

Juden und Jüdinnen stellten ab 1880 rund zehn Prozent der Wiener Gesamtbevölkerung. Dieser Wert blieb bis zum Ersten Weltkrieg im Wesentlichen konstant. ${ }^{1}$ Dabei wuchsen sowohl die Stadt insgesamt als auch die Anzahl der Juden und Jüdinnen in diesem Zeitraum rasant: 1880 lebten rund 73.200 Juden und Jüdinnen in Wien (Gesamtbevölkerung: 726.105), 1890 rund 118.500 (Gesamtbevölkerung: 1.728.738) und um 1900 rund 148.000 (Gesamtbevölkerung: 2.031.498). Im Jahr 1910 waren 175.318 Personen jüdischen Glaubens in Wien wohnhaft, das bedeutete einen Anteil von 8,6 Prozent an der Wiener Bevölkerung. ${ }^{2}$ Die in Wien ansässigen Juden und Jüdinnen stellten mit einem Anteil von rund 92 Prozent die überwiegende Mehrheit aller österreichischen Juden und Jüdinnen, in den (späteren) Bundesländern lebten nur knapp 15.000. Somit bildete die jüdische Bevölkerung im Jahr 1910 lediglich einen Anteil von 2,9 Prozent an der österreichischen Gesamtbevölkerung von 6.645.984 Personen (Gebietsstand: heutiges Österreich). ${ }^{3}$

1 Als Juden und Jüdinnen sind hier Personen mit mosaischem Glaubensbekenntnis gemeint. Zur NS-Definition eines Juden/einer Jüdin siehe weiter unten.

2 Eine Aufstellung der Wiener Bevölkerung nach Religionsbekenntnis seit 1869 findet sich im Textheft der Volkszählung 1934: Bundesamt für Statistik (Hg.), Die Ergebnisse der österreichischen Volkszählung vom 22. März 1934. Bundesstaat. Textheft (Statistik des Bundesstaates Österreich, Heft 1, Wien 1935) 50 f. Vgl. auch Hans Tietze, Die Juden Wiens, Geschichte - Wirtschaft - Kultur (Wien 1933, Reprint Wien 22008) 219; J[onas] Kreppel, Juden und Judentum von heute. Übersichtlich dargestellt. Ein Handbuch (Wien 1925) 348 f.; Statistische Zentralkommission (Hg.), Statistisches Handbuch für die Republik Österreich (1. Jahrgang, Wien 1920) 14.

3 Albert Lichtblau, Integration, Vernichtungsversuch und Neubeginn. Österreichisch-jüdische Geschichte 1848 bis zur Gegenwart. In: Eveline Brugger, Martha Keil, Albert Lichtblau, Christoph Lind, Barbara Staudinger, Geschichte der Juden in Österreich (Österreichische Geschichte, Ergänzungsband 4, Wien 2006) 447-563, hier 502; Jonny Moser, Die Katastrophe der Juden in Österreich 1938-1945. Ihre Voraussetzungen und ihre Überwindung. In: Der gelbe Stern in Österreich (Eisenstadt 1977), 67-133, hier 67.

○ Open Access. (C) 2019 Susanne Helene Betz, publiziert von De Gruyter. (cc)BY Dieses Werk ist lizenziert unter der Creative Commons Attribution 4.0 International Lizenz (CC BY 4.0). 
Rund die Hälfte der Wiener Juden und Jüdinnen lebte in der zweiten Hälfte des 19. Jahrhunderts in der Leopoldstadt. Damals umfasste dieser Stadtteil Wiens noch die gesamte Fläche zwischen Donaukanal und Donau; 1900 wurde sein nördlicher Teil, die Brigittenau, als eigenständiger Bezirk von der Leopoldstadt abgetrennt. Bereits im 17. Jahrhundert war Juden und Jüdinnen ein Teil der Leopoldstadt, der damals noch im Vorstadtbereich gelegene „Untere Werd“, als „Judenstadt“ bzw. Ghetto zugewiesen worden. ${ }^{4}$ Durch die Kontinuität dieses Siedlungsraums als Wohnort für Juden und Jüdinnen entwickelte sich dessen umgangssprachliche Bezeichnung „Mazzesinsel“ (nach dem während des jüdischen Pessachfestes verzehrten ungesäuerten Brot). Auf der „Mazzesinsel“ war es frommen Personen leichter möglich die Schabbat und Speisegesetze einzuhalten; ${ }^{5}$ Zuwandernde aus dem ländlichen Raum („Dorfjuden“), vor allem aber MigrantInnen aus Ungarn, Böhmen, Mähren und Schlesien sowie Galizien konnten Sprach- und Kulturbarrieren in dieser jüdisch geprägten Umgebung leichter überwinden. ${ }^{6}$ Im Jahr 1910 lebten in der Leopoldstadt und in der Brigittenau insgesamt 71.000 Juden und Jüdinnen; sie stellten dort 34 bzw. 14 Prozent der jeweiligen Wohnbevölkerung. ${ }^{7}$ Abgesehen von der „Mazzesinsel“ waren die Wohnorte der Juden und Jüdinnen vor allem auf die Wiener Innenstadt und den Alsergrund konzentriert. ${ }^{8}$

Die Volkszählung des Jahres 1910 weist - im Gegensatz zu den beiden Volkszählungen 1923 und 1934 - die Form der Erwerbstätigkeit der Bevölkerung nach Geschlecht und Religion aus. Dieses Zahlenmaterial ermöglicht die Aussage, dass sich zu Beginn des Jahrhunderts die Berufsstruktur der jüdischen Bevölkerung stark von jener der nichtjüdischen Bevölkerung unterschied: Jüdische Erwerbstätige waren deutlich häufiger selbstständig, unter den ArbeiterInnen hingegen unterrepräsentiert. Öfter waren sie auch in den

\footnotetext{
4 Barbara Staudinger, Die Zeit der Landjuden und der Wiener Judenstadt 1496-1670/71. In: Eveline Brugger et al., Geschichte der Juden in Österreich (Wien 2006) 229-338, hier $235 \mathrm{f}$. Zur Brigittenau vgl. Magistratsabteilung für Statistik (Hg.), Statistisches Jahrbuch der Stadt Wien für das Jahr 1929 (Neue Folge [im Folgenden: NF], 2. Jahrgang, Wien 1930) 1.

5 Ruth Burstyn, Die Geschichte des Leopoldstädter Tempels in Wien - 1858 bis 1938. In: Kairos 28 (1986) 228-249, hier 231 f. Die Angaben zur Wohnraumverteilung beziehen sich auf 1869.

6 Marsha Rozenblit, Die Juden Wiens 1867-1914. Assimilation und Identität (Forschungen zur Geschichte des Donauraumes 11, Wien/Köln/Graz 1989) $20 \mathrm{ff}$.

7 Leo Goldhammer, Die Juden Wiens. Eine statistische Studie (Wien 1927) 10. Zum Vergleich: In Floridsdorf lebten 1910 nur rund 1.800 Juden und Jüdinnen; sie stellten 2,28 Prozent der BezirkseinwohnerInnen.

8 Burstyn, Geschichte, 231 f.; Ruth Burstyn, Die „Schiffschul“ - Geschichte, Hintergründe. In: Historisches Museum der Stadt Wien (Hg.), „Heilige Gemeinde Wien“. Judentum in Wien. Sammlung Max Berger (Wien 1988) 45-50; Rozenblit, Juden, 80-105.
} 
Bereichen Handel und Verkehr bzw. Transportwesen sowie Industrie und Gewerbe und in freien Berufen tätig; die nichtjüdische Bevölkerung war im Gegensatz dazu sehr viel stärker in der Land- und Forstwirtschaft beschäftigt. ${ }^{9}$

Die starke Repräsentation von Juden und Jüdinnen in bestimmten Berufsfeldern ${ }^{10}$ hatte ihre Ursache in den historischen Limitierungen der jüdischen Bevölkerung hinsichtlich sozialer Handlungsspielräume und Berufswahl, die bis in das 19. Jahrhundert gültig waren (Gewerbeeinschränkungen für NichtChristen, Barrieren beim Zugang zu akademischen Berufen, Grundbesitzverbot etc. ${ }^{11}$ und wohl auch in der wichtigen Rolle religiöser Studien für jüdische Knaben und Männer. ${ }^{12}$ Letztlich brachte diese historische Entwicklung eine „modernere“ Berufsstruktur und eine höhere soziale Mobilität mit sich: „Kinder aus Bauern- und Arbeiterfamilien [konnten] in weitaus geringerem Ausmaß den höheren Bildungsweg anstreben [...] als Kinder aus Familien, die im Handel und Gewerbe, freien Beruf oder im Dienstleistungssektor" 13 tätig waren. Der Zulauf zu Bildungseinrichtungen seitens der Wiener jüdischen Bevölkerung lässt sich an der Hörerstatistik der zweiten Hälfte des 19. Jahrhunderts klar ablesen: 1889/90 stellten jüdische Studierende an der juridischen Fakultät der Universität Wien einen Anteil von 22 Prozent der Studierenden, an der medizinischen Fakultät 48 Prozent und an der philosophischen 15 Prozent. ${ }^{14}$ Von 681 Rechtsanwälten in Wien waren zum selben Zeitpunkt 394 jüdisch, die Mehrzahl stellten Juden auch unter den Ärzten. ${ }^{15}$ Diese Zahlen zeigen klar, dass der soziale Aufstieg jüdischer Familien ab der zweiten Hälfte des 19. Jahrhunderts in das Milieu der „Geldaristokratie“ und Intellektuellen des Wiener

9 Steven Beller, Wien und die Juden, 1867-1938 (Wien 1993) 57. Vgl. zu diesem Themenkomplex auch die Studie von Rozenblit, Juden, insb. 55 ff., sowie: K.u.k. Statistische Zentralkommission (Hg.), Die Ergebnisse der Volkszählung vom 31. Dezember 1910 (NF, 1. Band, 2. Heft, Wien 1914) 36, 54 f.; Lichtblau, Integration, 479 f., 461, 474 (zur Bevölkerungsverteilung); Kurt Schubert, Der Weg zur Katastrophe. In: Der gelbe Stern in Österreich, 31-66, hier 39 f.; Moser, Voraussetzungen, 68 f.; Goldhammer, Juden Wiens, $13 \mathrm{f}$.

10 Vgl. zur Diskussion derselben: Beller, Wien, 53 ff.; Lichtblau, Integration, 482 ff.; Rozenblit, Juden, insb. $55 \mathrm{ff}$.

11 Den Erwerb von Liegenschaften durch Juden und Jüdinnen gewährte erst die kaiserliche Verordnung vom 18. Februar 1860; das Ende der Einschränkungen im Gewerbe trat durch den Wegfall der alten Zunftordnung 1859 ein. Vgl. dazu auch Michael Wladika, Hitlers Vätergeneration. Die Ursprünge des Nationalsozialismus in der k. u. k. Monarchie (Wien 2005) 46 f. Gänzliche rechtliche Gleichstellung und freien Zugang zu bestimmten akademischen Berufen ermöglichte schlussendlich das Staatsgrundgesetz vom 21. Dezember 1867.

12 Lichtblau, Integration, $481 \mathrm{f}$.

13 Lichtblau, Integration, $482 \mathrm{f}$.

14 Tietze, Juden, 220. Vgl. auch Wladika, Vätergeneration, 46.

15 Tietze, Juden, 220; Wladika, Vätergeneration, 46. 
Fin de Siècle und ihr Eintritt in Besitz- und Bildungsbürgertum neben staatlicher Liberalisierung, Nobilitierungen und aktivem Kulturschaffen vorrangig durch Bildung bewirkt wurde. ${ }^{16}$

\section{3 bis 1926}

Bei der Volkszählung 1923 wurden 201.513 Personen jüdischen Glaubens in Wien gezählt. Sie bildeten 91,5 Prozent der jüdischen Bevölkerung Österreichs (insgesamt: 220.208) ${ }^{17}$ und 10,8 Prozent aller 1.865.780 WienerInnen. Damit formten Personen jüdischen Glaubens in Wien die zweitstärkste Religionsgruppe nach den KatholikInnen. ${ }^{18}$ Insgesamt stellte die jüdische Bevölkerung in Österreich (Gesamtbevölkerung: 6.534.481) ${ }^{19}$ im Jahr 1923 einen Anteil von 3,37 Prozent. Bis 1926 nahm die Zahl der jüdischen EinwohnerInnen Wiens weiter zu; zu keinem späteren Zeitpunkt lebten mehr Juden und Jüdinnen in der Hauptstadt.

Innerhalb Wiens verteilte sich die jüdische Bevölkerung im Jahr 1923 wie folgt (siehe Tabelle 1): In acht Bezirken Wiens stellten jüdische BürgerInnen mehr als zehn Prozent der BezirkseinwohnerInnen. Die größte Gruppe der Wiener Juden und Jüdinnen lebte weiterhin in der Leopoldstadt, ${ }^{20}$ wo sie beinahe zwei Fünftel der Bezirksbevölkerung stellten. In diesem Bezirk war auch die jüdische Infrastruktur am dichtesten ausgeprägt. Im Alsergrund und der Inneren Stadt bildeten Juden und Jüdinnen ein Viertel, in der an die Leopoldstadt grenzenden Brigittenau beinahe ein Fünftel und in Mariahilf, Neubau, Josef-

16 Peter Pulzer, Nachwort. In: Hans Tietze, Die Juden Wiens. Geschichte - Wirtschaft - Kultur (Wien 2008) 290-310, hier 293-302. Vgl. dazu auch: Steven Beller, Was nicht im Baedeker steht. Juden und andere Österreicher im Wien der Zwischenkriegszeit. In: Stern, Eichinger (Hg.), Wien und die jüdische Erfahrung, 1-16, hier 3 ff., und Steven Beller, Antisemitism. A Very Short Introduction (Oxford 2007) 38, sowie Beller, Wien, $72 \mathrm{ff}$.

17 Leo Goldhammer, Von den Juden Österreichs. In: Taubes, Bloch (Hg.), Jahrbuch, 6-16, hier 8.

18 Goldhammer, Juden Wiens, 11. KatholikInnen stellten 1923 81,4 Prozent und 1934 78,7 Prozent der Wiener Bevölkerung; die drittstärkste Gruppe bildeten mit rund fünf Prozent die ProtestantInnen. Magistratsabteilung für Statistik (Hg.), Statistisches Jahrbuch der Stadt Wien 1930-1935 (NF, 3. Band, Wien o. J.) 12; Bundesamt für Statistik, Volkszählung 1934, Textheft, $45 \mathrm{f}$.

19 Bundesamt für Statistik (Hg.), Statistisches Handbuch für die Republik Österreich, 11. Jahrgang (Wien 1930) 2.

20 Die Leopoldstadt lag laut Volkszählung 1923 mit 155.152 BewohnerInnen an zweiter Stelle nach dem bevölkerungsreichsten Bezirk Ottakring; auch in ihrer flächenmäßigen Ausdehnung lag die Leopoldstadt (nach Hietzing) an zweiter Stelle. Magistratsabteilung für Statistik, Jahrbuch 1930-1935, 4, 7. 
stadt und Döbling schließlich deutlich mehr als ein Zehntel der Bezirksbevölkerung. In den vier (erstgereihten) Bezirken lebten somit rund 60 Prozent, in insgesamt acht Bezirken insgesamt 75 Prozent der Wiener Juden und Jüdinnen. Damit lebte diese Minderheit in deutlich konzentrierteren Räumen der Stadt als andere Bevölkerungsgruppen wie etwa die Wiener TschechInnen. ${ }^{21}$

Tab. 1: Verteilung der jüdischen Bevölkerung Wiens auf die 21 Wiener Bezirke im Jahr 1923, Absolutzahlen und Anteil an der jeweiligen Bezirksbevölkerung in Prozent, basierend auf der Volkszählung 1923. ${ }^{22}$

\begin{tabular}{lc}
\hline Bezirk - Bezirksname & $\begin{array}{c}\text { Jüdische Bevölkerung im Bezirk und Anteil } \\
\text { an der Bezirksbevölkerung in Prozent }\end{array}$ \\
\hline 1 - Innere Stadt & $10.462(24,3 \%)$ \\
2 - Leopoldstadt & $59.722(38,5 \%)$ \\
3 - Landstraße & $14.204(9,83 \%)$ \\
4 - Wieden & $5.570(9,66 \%)$ \\
5 - Margareten & $4.471(4,97 \%)$ \\
6 - Mariahilf & $8.941(16,4 \%)$ \\
7 - Neubau & $9.838(15,6 \%)$ \\
8 - Josefstadt & $6.932(13,8 \%)$ \\
9 - Alsergrund & $23.746(25,1 \%)$ \\
10 - Favoriten & $4.011(2,8 \%)$ \\
11 - Simmering & $400(0,9 \%)$ \\
12 - Meidling & $2.405(2,5 \%)$ \\
13 - Hietzing & $5.977(4,6 \%)$ \\
14 - Rudolfsheim & $3.706(4,7 \%)$ \\
15 - Fünfhaus & $2.904(5,6 \%)$ \\
16 - Ottakring & $4.881(3,1 \%)$ \\
17 - Hernals & $3.829(4,3 \%)$ \\
18 - Währing & $4.601(5,4 \%)$ \\
19 - Döbling & $5.665(10,2 \%)$ \\
20 - Brigittenau & $17.572(18,0 \%)$ \\
21 - Floridsdorf & $1.676(2,0 \%)$ \\
\hline
\end{tabular}

Die jüdische Bevölkerungsgruppe Wiens bildete trotz ihrer räumlichen Konzentration auf einige bestimmte Stadtteile eine sehr heterogene und diverse Personengruppe. Sie setzte sich vorrangig aus ,assimilationsorientierten“, kaum zio-

21 Albert Lichtblau, Partizipation und Isolation. Juden in Österreich in den „langen“ 1920er Jahren. In: Archiv für Sozialgeschichte 37 (1977) 231-254, hier 233; Eckdaten zu weiteren Minderheiten finden sich in Magistratsabteilung für Statistik, Jahrbuch 1930-1935, 12.

22 Kreppel, Juden, 710; Goldhammer, Juden Wiens, 10. 
nistisch eingestellten Juden und Jüdinnen, aus religiös orthodoxen Gruppen aus Ungarn, Böhmen und Mähren sowie den „Ostjuden“ aus Galizien und der Bukowina zusammen. Daneben existierte in Wien unter anderem noch die „türkische Gemeinde“, eine Gruppe sephardischer Juden und Jüdinnen. ${ }^{23}$ Die Heterogenität der jüdischen Bevölkerung Wiens spiegelte sich auch in der Vielfalt der innerhalb der Israelitischen Kultusgemeinde (IKG) Wien auftretenden politischen Parteien wider. Die IKG Wien war gemäß Israelitengesetz von 1890 die für die Verwaltung des religiösen Lebens der in ihrem Sprengel wohnhaften Juden und Jüdinnen zuständige Einrichtung. ${ }^{24}$ Das politische Spektrum reichte von orthodoxen, sowohl antizionistischen als auch zionistischen Gruppen über nichtreligiöse Assimilierte zu stark jüdisch-national orientierten, zionistischen Gruppierungen. Auch in Fragen der gelebten Religiosität unterschieden sich die Wiener Juden und Jüdinnen stark voneinander: Orthodoxe aus Ungarn, Böhmen und Mähren fanden ihr religiöses Zentrum vielfach in der Wiener „Schiffschul“ in der Leopoldstadt. Die „Ostjuden“ betrieben ein Bethaus nach polnischem Ritus in der Leopoldgasse 29, ebenfalls im 2. Wiener Gemeindebezirk. „Assimilierungsorientierte“ Juden und Jüdinnen fühlten sich eher der Kultusgemeinde in der Seitenstettengasse in Wien, Innere Stadt, zugehörig, während die SephardInnen einen Tempel im orientalischen Stil in der Zirkusgasse in Wien Leopoldstadt nutzten. ${ }^{25}$ Zudem existierten zahlreiche weitere, vereinsmäßig betriebene Bethäuser und Synagogen mit teilweise unterschiedlichem Ritus. ${ }^{26}$ Streng orthodoxe und chassidische Juden und Jüdinnen waren jedoch in der Minderheit, ein hoher Prozentsatz der jüdischen Bevölkerung Wiens Lichtblau schätzt sogar einen Anteil von rund der Hälfte - besuchte selbst zu den hohen jüdischen Feiertagen keinen Tempel und lebte in der ersten Hälfte des 20. Jahrhunderts weitgehend areligiös. ${ }^{27}$ Weiters gestaltete sich das jüdische Leben Wiens abseits der zahlreichen religiösen Vereine auch aufgrund einer Vielzahl von Vereinigungen für wohltätige Zwecke, Frauenvereinen, Jugendgruppen, Sportklubs und zionistischen Vereinen sehr heterogen. ${ }^{28}$

23 Kreppel, Juden, 711; Lichtblau, Integration, 495, 499; Bericht des Präsidiums und des Vorstandes der Israelitischen Kultusgemeinde Wien über die Tätigkeit in den Jahren 1933-1936 (Wien 1936), 109.

24 Vgl. Israelitengesetz vom 21. März 1890, RGBl 57/1890; Lichtblau, Integration, 458.

25 Kreppel, Juden, 710 ff.; Lichtblau, Partizipation, 240; Moser, Voraussetzungen, 67-109.

26 Vgl. etwa Löbel Taubes, Chaim Bloch (Hg.), Jüdisches Jahrbuch für Österreich (Wien 5393 [1932/33]) 33-51; Magistratsabteilung für Statistik, Jahrbuch 1930-1935, 204.

27 Lichtblau, Partizipation, 240.

28 Vgl. die Aufstellungen in Taubes, Bloch, Jahrbuch, 51 ff., in den Berichten der Israelitischen Kultusgemeinde aus der Zwischenkriegszeit, etwa Bericht, 1933-1936, 41-44; Shoshana Duizend-Jensen, Jüdische Gemeinden, Vereine, Stiftungen und Fonds. „Arisierung“ und Restitu- 
Rund die Hälfte (57,7 Prozent) der jüdischen WienerInnen war laut Volkszählung 1923 im Ausland geboren. Diese Personen stammten vorwiegend aus Galizien, der Bukowina und Ungarn. ${ }^{29}$ Allein in der Leopoldstadt lag der Anteil der im Ausland geborenen Bevölkerung an der gesamten Bezirksbevölkerung bei 44 Prozent. ${ }^{30}$ Rund 25.000 vorrangig männliche Juden waren in den Jahren nach dem Ersten Weltkrieg als Flüchtlinge aus Galizien und der Bukowina nach Wien gekommen, ${ }^{31}$ was für die Zwischenkriegszeit zu einem geringeren Frauenanteil unter den Wiener Juden und Jüdinnen im Vergleich zur sonstigen Bevölkerung führte. ${ }^{32}$ Von den jüdischen WienerInnen nutzen allerdings nur erstaunlich wenige als Umgangssprache das Jiddische, laut Volkszählung 1923 handelte es sich um insgesamt 2.434 vorwiegend aus Galizien oder der Bukowina stammende Personen, die mehrheitlich in der Leopoldstadt, in der Brigittenau und im Alsergrund lebten. ${ }^{33}$ Es waren vorwiegend diese oft als „Ostjuden“ bezeichneten, zum Teil Jiddisch sprechenden und $\mathrm{zu}$ den ärmeren Schichten der Wiener Bevölkerung zählenden Juden und Jüdinnen, die wiederholt große Ablehnung der sich der deutschen Kultur verbundenen „Westjuden“ erfuhren. ${ }^{34}$

Der starke Trend zur Bildung innerhalb der Wiener Juden und Jüdinnen hatte sich auch nach dem Ersten Weltkrieg fortgesetzt: Jüdische StudentInnen nahmen im Jahr 1924 mit fast einem Drittel aller Studierenden (30 Prozent) einen weiterhin sehr hohen Anteil aller HochschülerInnen ein, wenngleich ihr Anteil 1920/21 höher gewesen war (42 Prozent) ${ }^{35}$ und bis 1938 tendenziell sank. ${ }^{36}$ Die Studienschwerpunkte jüdischer Studierender lagen wie Ende des 19. Jahrhunderts bei den Rechtswissenschaften (36 Prozent aller Studierenden

tion (Veröffentlichungen der Österreichischen Historikerkommission 21/2, Wien/München 2004) 38-50 sowie Kapitel 4.

29 Hingegen war nur rund ein Viertel der katholischen EinwohnerInnen Wiens im Ausland geboren. Lichtblau, Integration, 500 f.; Bundesamt für Statistik (Hg.), Statistisches Handbuch für die Republik Österreich, 6. Jahrgang (Wien 1925) 14.

30 Bundesamt für Statistik, Handbuch 1925, 14.

31 Lichtblau, Integration, 490 f., 501; Goldhammer, Juden Wiens, 13, 25 f.; Lichtblau, Partizipation, $234 \mathrm{f}$.

32 Lichtblau, Partizipation, 243; Goldhammer, Juden Wiens, 25; Magistratsabteilung für Statistik, Jahrbuch 1930-1935, 8; Bundesamt für Statistik, Volkszählung 1934, Textheft, 28 ff.

33 Lichtblau, Integration, 497; Lichtblau, Partizipation, 233. Nur ein Drittel dieser Personen war in Wien geboren. Magistratsabteilung für Statistik, Jahrbuch 1929 (NF, 2. Jahrgang) 6. 1934 gaben nur noch 510 Personen Jiddisch als Umgangssprache an. Magistratsabteilung für Statistik, Jahrbuch 1930-1935, 13; Bundesamt für Statistik, Volkszählung 1934, Textheft, 53.

34 Lichtblau, Partizipation, 239; Lichtblau, Integration, $476 \mathrm{f}$.

35 Tietze, Juden, 259 f.; Goldhammer, Juden Wiens, 39.

36 Maderegger, Ständestaat, $152 \mathrm{ff}$. 
im Studienjahr 1920/21; 25 Prozent im Studienjahr 1924/25), der Medizin (53 Prozent im Studienjahr 1920/21; 34 Prozent im Studienjahr 1924/25) und bei Fächern der philosophischen Fakultät (37 Prozent aller Studierenden im Studienjahr 1920/21; 22 Prozent im Studienjahr 1924/25) ${ }^{37}$ sowie auf Studienfächern der Hochschule für Welthandel (33 Prozent im Studienjahr 1920/21; 41 Prozent aller Studierenden 1923/24). ${ }^{38}$ Die Technische Hochschule und die Universität für Bodenkultur verzeichneten deutlich weniger jüdische Studierende (20 resp. zwei Prozent im Studienjahr 1924/25), an der veterinärmedizinischen Hochschule und der Akademie der bildenden Künste lagen ihre Anteile zu Beginn der 1920er-Jahre lediglich im einstelligen Bereich. ${ }^{39}$ Jüdische Studentinnen waren nach der 1897 stufenweise beginnenden Öffnung der Universitäten - die Zulassung von Frauen war an der philosophischen Fakultät ab 1897, an der medizinischen ab 1900 und an der rechts- und staatswissenschaftlichen ab 1919 gestattet - grundsätzlich in allen genannten Studienrichtungen vertreten. ${ }^{40}$

\section{Wendepunkt 1927}

Ab 1927 sank die Zahl der Wiener Juden und Jüdinnen kontinuierlich. Dieser Rückgang der jüdischen Bevölkerung hatte mehrere Ursachen, wurzelte aber zunächst vorrangig in der bereits seit den 1880er-Jahren verstärkten Konversionsbewegung, ausgelöst durch den zunehmenden Antisemitismus, der etwa Karrierechancen minderte. ${ }^{41}$ Anna L. Staudacher spricht von rund 18.000 Personen, davon 10.000 Männer, die im Zeitraum von 1868 bis 1914 aus der IKG Wien austraten. ${ }^{42}$ In den statistischen Gesamtzahlen der jüdischen Bevölkerung machte sich dieser Trend aufgrund der vielen jüdischen Flüchtlinge des Ersten Weltkriegs erst verspätet bemerkbar. ${ }^{43}$ Die Austrittszahlen wurden ins-

37 Goldhammer, Juden Wiens, 40.

38 Goldhammer, Juden Wiens, 42.

39 Goldhammer, Juden Wiens, $41 \mathrm{f}$.

40 Magistratsabteilung für Statistik, Jahrbuch 1930-1935, 178 ff.; Katharina Kniefacz, Frauen an der Universität Wien 1878-2015, online unter: http://geschichte.univie.ac.at/de/themen/ frauen-der-universitat-wien (November 2017).

41 Rozenblit, Juden, 137-151; Lichtblau, Integration, 504; Goldhammer, Juden Wiens, $33 \mathrm{f}$.

42 Anna L. Staudacher, ,.... meldet den Austritt aus dem mosaischen Glauben“. 18.000 Austritte aus dem Judentum in Wien, 1868-1914: Namen - Quellen - Daten (Frankfurt/M. 2009) 11.

43 Tietze, Juden, 260; Lichtblau, Integration, 501-504. Vgl. auch Sylvia Maderegger, Die Juden im österreichischen Ständestaat. 1934-1938 (Wien 1973) 60-66; Goldhammer, Juden Wiens, 2934; Moser, Voraussetzungen, $68 \mathrm{ff}$., sowie Lichtblau, Partizipation, $234 \mathrm{f}$. 
besondere dadurch gesteigert, da Juden und Jüdinnen in Wien - im Gegensatz $\mathrm{zu}$ anderen europäischen Städten wie etwa Berlin - zum einen ihre Kinder nur dann taufen lassen konnten, wenn sich auch ein Elternteil taufen ließ, ${ }^{44}$ und zum anderen Ehen zwischen Angehörigen der christlichen und jüdischen Religion in Österreich nicht erlaubt waren. Das Ehehindernis wegen Religionsverschiedenheit gemäß $\S 64 \mathrm{ABGB}^{45}$ erzwang vor Eheschließungen daher den Übertritt in die Religion des Partners bzw. der Partnerin oder zumindest einen Austritt und die Erklärung der Konfessionslosigkeit. ${ }^{46}$ In letzteren Fällen war seit 1868 wenigstens das Eingehen einer amtlich geschlossenen Notzivilehe möglich. ${ }^{47}$

Das Ehehindernis gemäß $\S 64$ konnte zwar auch durch einen beim jeweils zuständigen Landeshauptmann anzusuchenden Dispens nachgesehen werden, was eine Heirat vor den politischen Behörden ohne Austritt aus den jeweiligen Religionsgemeinschaften ermöglichte, ${ }^{48}$ es handelte sich dabei hinsichtlich Ehen zwischen KatholikInnen und Juden bzw. Jüdinnen aber um eine insgesamt wenig gängige Praxis. ${ }^{49}$ Da auch das kanonische Recht solche Ehen aus-

44 Lichtblau, Partizipation, 237.

$45 \S 64$ ABGB: „Eheverträge zwischen Christen und Personen, welche sich nicht zur christlichen Religion bekennen, können nicht gültig eingegangen werden." Diese Norm ersetzten ab August 1938 die Bestimmungen des deutschen Reichsgesetzblatts (dRGBl) I 1938, $807 \mathrm{ff}$.

46 Lichtblau, Integration, 503; Goldhammer, Juden Wiens, 17-19.

47 RGBl Nr. 47 vom 15. Mai 1868, Art. II Abs. 1; Lichtblau, Partizipation, 237.

48 Vgl. Josef Schey (Hg.), Das Allgemeine bürgerliche Gesetzbuch [...] mit einer Übersicht der Rechtsprechung des Obersten Gerichtshofes (Wien $\left.{ }^{23} 1936\right) 45$ (Anm. 8; § 64), 52 (§ 83) und Erkenntnisse des österr. Obersten Gerichtshofes, Bd. 6 (Wien 1925) 167 ff., Entscheidung Nr. 73 vom 21. 2. 1924 (beides mit weiteren Verweisen); Österreichische Zeitschrift für Verwaltung (8. 11. 1917) 184; Österreichische Anwalts-Zeitung (1934) 358.

49 Zur Anzahl der Fälle, in denen derartige Dispense zwischen 1868 und 1938 erteilt wurden, liegen keine Verlaufszahlen vor. Der staatliche Dispens scheint für jüdisch-christliche Ehen vor 1900 kaum, nach 1918 mit zunehmender Häufigkeit erteilt worden sein. Vgl. Juristische Blätter ([JBl] 1906) 366 f., 413 f.; Österreichische Zeitschrift für Verwaltung (8. 11. 1917) 184; Neue Freie Presse (8. 7. 1925) 3; JBl (1936) 299f. Die amtlichen Statistiken weisen für Wien im Jahr 1920 241 (von insgesamt 30.137), im Jahr 192884 (von insgesamt 16.137) und im Jahr 1935156 (von insgesamt 13.291) Eheschließungen als Verbindungen eines katholischen mit einem jüdischen Partner aus. Allerdings geht aus diesen Zahlen weder der Anteil ausländischer Staatsangehöriger unmittelbar hervor, noch, ob zu den Ehen nur ein staatlicher oder auch ein kirchlicher Dispens vorlag. Bundesamt für Statistik (Hg.), Die Bewegung der Bevölkerung in den Jahren 1914 bis 1921 (Wien 1923) 112f.; Bundesamt für Statistik, Handbuch 1930, 24; Magistratsabteilung für Statistik, Jahrbuch 1930-1935, 48 f. Durchschnittlich wurden in Wien zwischen 1926 und 1928 pro Jahr 310, zwischen 1930 und 1935 pro Jahr 516 Dispense vom Ehehindernis der Religionsverschiedenheit gemäß § 64 ABGB erteilt (insgesamt, d. h. alle fraglichen Glaubensbekenntnisse). Magistratsabteilung für Statistik (Hg.), Statistisches Jahrbuch der Stadt Wien 1929 (NF, 1. Jahrgang, Wien o. J.) 9; Magistratsabteilung für Statistik, Jahrbuch 1930-1935, 50. 
schloss (Can. 1086 § 1), war für eine kirchliche Trauung zweier Personen mit diesen Religionsbekenntnissen zudem ein entsprechender Dispens der katholischen Kirche notwendig. ${ }^{50}$

Zwischen 1920 und 1930 traten jährlich rund 1.000 Personen und mehrheitlich Frauen - sowohl für Eheschließungen aber wohl auch aus politischen und/oder emanzipatorischen Gründen - aus der IKG Wien aus. ${ }^{51}$ Nach 1930 bewegten sich die Austritte auf etwas niedrigerem Niveau und lagen bei rund $800 \mathrm{im}$ Jahr 1931 und etwa $500 \mathrm{im}$ Jahr $1935 .^{52}$ Insgesamt handelte es sich um weitere rund 18.000 Personen, die nach Ende des Ersten Weltkrieges bis zum „Anschluss“ 1938 austraten. ${ }^{53}$ Beinahe die Hälfte der Ausgetretenen schloss sich der katholischen Kirche an, rund ein Viertel der evangelischen, der Rest blieb konfessionslos. ${ }^{54}$ Neben den Austritten gelten ein allgemeiner Geburtenrückgang ${ }^{55}$, eine erhöhte Sterblichkeit durch Überalterung und eine negative Wanderungsbilanz - d.h. Abwanderung aus Wien, vorrangig in die USA und nach Palästina - zu den Gründen des jüdischen Bevölkerungsrücklaufs seit $1927 .{ }^{56}$

50 Norbert Lüdecke, § 86. Die rechtliche Ehefähigkeit und die Ehehindernisse. In: Stephan Haering, Wilhelm Rees, Heribert Schmitz (Hg.), Handbuch des katholischen Kirchenrechts (Regensburg ${ }^{32015)}$ 1282-1314. Im Judentum gibt es den (für KatholikInnen auch heute noch notwendigen) Dispens dieser Art nicht, interreligiöse Heiraten gelten als ungültig. Erich Rosenthal, Ben-Zion Schereschewsky, Mervin Verbit, Sergio DellaPergola, Mixed Marriage, Intermarriage. In: Michael Berenbaum, Fred Skolnik (Hg.), Encyclopaedia Judaica, Band 14 (Detroit ${ }^{2} 2007$ ) 373-385.

51 Lichtblau, Integration, 504; Goldhammer, Juden Wiens, 7, 33 f., 65. Zu den Austrittsmotiven von Frauen vgl. auch Michaela Raggam-Blesch, „Being different where being different was definitely not good.“ Identitätskonstruktionen jüdischer Frauen in Wien. In: Stern, Eichinger (Hg.), Wien und die jüdische Erfahrung, 257-276; Staudacher, Austritt, 11. Vgl. auch Magistratsabteilung für Statistik, Jahrbuch 1929, (NF, 1. Jahrgang) 40 f.; Bundesamt für Statistik (Hg.), Statistisches Handbuch für den Bundesstaat Österreich, 17. Jahrgang (Wien 1937) 25; Bericht, 1933-36, 109.

52 Magistratsabteilung für Statistik, Jahrbuch 1930-1935, 204.

53 Philomena Leitner, Assimilation, Antisemitismus und NS-Verfolgung. Austritte aus der Jüdischen Gemeinde in Wien 1900-1944 (Diss. Universität Wien 2003) 152.

54 Goldhammer, Juden Wiens, 31. Das Phänomen des Austritts aus der Religionsgemeinschaft war in den 1920er-Jahren jedoch kein spezifisch jüdisches; auch die katholische Religionsgemeinschaft war mit Austrittsbewegungen konfrontiert. Lichtblau, Partizipation, 237 f.; Bundesamt für Statistik, Volkszählung 1934, Textheft, 49, 51 f.; vgl. auch Bundesamt für Statistik (Hg.), Statistisches Handbuch für den Bundesstaat Österreich, 15. Jahrgang (Wien 1935) 36.

55 Eine Aufstellung über mehrere Jahre findet sich im Bericht, 1933-1936, 109; Lichtblau, Partizipation, 234-238, insb. 236.

56 Lichtblau, 501 ff.; Lichtblau, Partizipation, 235 f.; Maderegger, Ständestaat, 2; Jonny Moser, Demographie der jüdischen Bevölkerung Österreichs: 1938-1945 (Dokumentationsarchiv des Österreichischen Widerstandes, Wien 1999) 11, 22; Goldhammer, Juden Wiens, 15 ff. 


\section{4 bis 1938}

1934 lebten in Wien 176.034 Personen israelitischer Religion; sie stellten einen Anteil von 9,4 Prozent der insgesamt 1.874.130 WienerInnen. ${ }^{57}$ Die Wiener Juden und Jüdinnen bildeten 91 Prozent aller jüdischen EinwohnerInnen (insgesamt 191.481) und stellten 2,8 Prozent der österreichischen Gesamtbevölkerungszahl von 6.760 .233 .58

Die räumliche Verteilung der Wiener Juden und Jüdinnen entsprach 1934 weitgehend jener von 1923 und hatte sich, abgesehen von einem leichten Trend der Abwanderung in die Stadtrandbezirke, ${ }^{59}$ kaum verändert: Weiterhin war der 2. Wiener Gemeindebezirk mit 34 Prozent jüdischen BewohnerInnen vor der Inneren Stadt und dem Alsergrund mit 24 bzw. 23 Prozent jüdischem Bevölkerungsanteil der jüdische Bezirk Wiens. ${ }^{60}$ In der Leopoldstadt befand sich auch etwas mehr als die Hälfte der insgesamt sechs Tempel und 89 Bethäuser der IKG (44), nur 14 dieser Einrichtungen waren im zweitgereihten Bezirk Brigittenau, jeweils sieben im 8. und 9. Bezirk zu finden. ${ }^{61} \mathrm{Zusätzlich} \mathrm{zu}$ den genannten Stätten gab es in Wien 193454 jüdische Bibel- und Hebräischschulen und 116 jüdische religiöse Vereine. ${ }^{62}$

Juden und Jüdinnen waren 1934 auch unverändert in den oben bereits genannten Studienrichtungen der Universität Wien überrepräsentiert, so etwa unter den Jusstudierenden (Sommersemester 1933: 16 Prozent, Wintersemester 1936/37: 10 Prozent), den angehenden MedizinerInnen (32 bzw. 25 Prozent) und den Studierenden der philosophischen Fakultät (jeweils 12 Prozent). ${ }^{63}$ Zahlrei-

57 Bundesamt für Statistik (Hg.), Die Ergebnisse der österreichischen Volkszählung vom 22. März 1934. Bundesstaat. Tabellenheft (Statistik des Bundesstaates Österreich, Heft 2, Wien 1935), 402 f. Vgl. weiter Maderegger, Ständestaat, 1; Lichtblau, Partizipation, 3; Österreichisches Statistisches Landesamt (Hg.), Statistisches Jahrbuch für Österreich 1938 (Wien 1938) 17.

58 Karl Stuhlpfarrer, Judenfeindschaft und Judenverfolgung in Österreich seit dem Ersten Weltkrieg. In: Anna Drabek, Wolfgang Häusler, Kurt Schubert, Karl Stuhlpfarrer, Nikolaus Vielmetti (Hg.), Das österreichische Judentum (Wien 1988) 141-236, hier 155; Bericht, 1933-1936, 109; Statistisches Landesamt, Jahrbuch 1938, 17.

59 Bundesamt für Statistik, Volkszählung 1934, Textheft, 17, 25.

60 Lichtblau, Partizipation, 234; Bundesamt für Statistik, Volkszählung 1934, Tabellenheft, 3. 61 Bericht, 1933-1936, 32. Weitere Privat- und Vereinsbethäuser waren im 2., 3., 7., und 20. Bezirk eingerichtet.

62 Magistratsabteilung für Statistik, Jahrbuch 1930-1935, 204. Vgl. dazu jeweils auch die Berichte der Israelitischen Kultusgemeinde sowie Taubes, Bloch, Jahrbuch, $32 \mathrm{ff}$.

63 Maderegger, Ständestaat, 153. Die Absolutzahlen für das Studienjahr 1933/34 (mit etwas reduzierten Zahlen jüdischer Studierender im Sommersemester 1934) finden sich im Bundesamt für Statistik, Handbuch 1935, 212 f. bzw. Magistratsabteilung für Statistik, Jahrbuch 19301935, 178. 
che der deutsch-nationalen und antisemitisch eingestellten Burschenschaften forderten einen Numerus clausus für jüdische HochschülerInnen. ${ }^{64}$ An den weiteren Universitäten in Wien wiesen nur die Studienrichtungen der Technischen Hochschule mit einem durchschnittlichen Anteil jüdischer Studierender von rund elf Prozent in den Sommersemestern 1933 und 1936, die Wiener Hochschule für Welthandel mit einem Anteil von rund 18 bzw. 15 Prozent und die Fachschule für Musik und darstellende Kunst mit einem Anteil von rund 20 Prozent (im Sommersemester 1936) zweistellige Prozentwerte jüdischer Studierender auf. ${ }^{65}$

Diesen Studierendenzahlen entsprechend waren Juden und Jüdinnen in den (freien) Rechtsberufen, als ÄrztInnen und als JournalistInnen mit über 20 Prozent fast doppelt so oft tätig wie die sonstige erwerbstätige Bevölkerung. Dies zeigen für die Mitte der 1930er-Jahre erstellte Daten des Statistikers Leo Goldhammer ${ }^{66}$, die in den Tabellen 2 und 3 auszugsweise ersichtlich sind: Juden und Jüdinnen stellten etwa 62 Prozent bzw. 63 Prozent aller selbstständigen RechtsanwältInnen bzw. ZahnärztInnen sowie 47 Prozent der ÄrztInnen. Frauen waren in allen Berufssparten der freien Berufe mit Ausnahme der Notare und Tierärzte vertreten; ${ }^{67} \mathrm{im}$ (knapp) zweistelligen Bereich lagen ihre Prozentanteile jedoch nur bei den ÄrztInnen (9 Prozent), ZahnärztInnen (13 Prozent) und ApothekerInnen (19 Prozent). ${ }^{68}$ Als Angestellte waren Juden und Jüdinnen im Bankenbereich und unter Universitätsprofessoren ${ }^{69}$ überproportional oft zu finden. Im öffentlichen Dienst gab es hingegen kaum jüdische VertreterInnen, weder in den juristischen Berufen noch in öffentlichen Krankenanstalten. Diese Karrierewege waren in der Regel aus Gründen des Antise-

64 Maderegger, Ständestaat, 152 f., 156.

65 Bundesamt für Statistik, Handbuch 1935, 214.

$66 \mathrm{Zu}$ Goldhammer vgl. Österreichisches Biographisches Lexikon 1815-1950, Bd. 2, Lfg. 6 (Wien 1957) 23.

67 Alexander Mejstrik, Therese Garstenauer, Peter Melichar, Alexander Prenninger, Christa Putz, Sigrid Wadauer, Berufsschädigungen in der nationalsozialistischen Neuordnung der Arbeit: vom österreichischen Berufsleben 1934 zum völkischen Schaffen 1938 - 1940 (Veröffentlichungen der Österreichischen Historikerkommission 16, Wien/München 2004) 159f. Frauen waren ab 1919 zum tierärztlichen Studium zugelassen; 1939 schloss die erste Frau dieses Studium ab. Vgl.: O. V., Die Veterinärmedizinische Universität Wien feiert ihren 250. Geburtstag, online unter: https://www.vetmeduni.ac.at/de/infoservice/presseinformationen/presseinfo2015/ 250-vetmedunivienna/ (Mai 2017).

68 Mejstrik et al., Berufsschädigungen, 159 f. Zahlen für die Gesamtbevölkerung laut Volkszählung 1934.

69 Die erste ordentliche Professur an eine Frau verlieh die Universität Wien im Jahr 1956. Ab 1921 war die Jüdin Elise Richter Inhaberin einer außerordentlichen Professur. Kniefacz, Frauen. 
mitismus verwehrt bzw. wurden ab 1934 weiter erschwert. ${ }^{70}$ Für 1934 nennt Goldhammer von den 42.000 Angestellten der Stadt Wien nur 142 jüdische (0,34 Prozent), und im Bundesdienst bildeten die 682 jüdischen Bediensteten nur den Anteil von 0,42 Prozent aller 160.700 österreichweit Angestellten. ${ }^{71}$ Im Jahr 1937 stellten Juden und Jüdinnen in Wien nur mehr 0,25 Prozent der städtischen Angestellten (inkl. LehrerInnen). ${ }^{72}$

Überproportional vertreten war die jüdische Bevölkerung Wiens in den 1930er-Jahren - basierend auf Angaben Leo Goldhammers - weiterhin im Handel und Verkehr: Dort waren 1934 mit 55 Prozent mehr als doppelt so viele jüdische Erwerbstätige tätig als in der Restbevölkerung. Es gab einige Handelssegmente, in denen jüdische Erwerbstätige besonders häufig vertreten waren, so stellten Juden und Jüdinnen 74 Prozent der WeinhändlerInnen, 73 Prozent der TextilhändlerInnen, 61 Prozent der SpirituosenhändlerInnen, und bildeten weiters im Benzin-/Öl-Handel und im Schuhhandel mit 54 Prozent bzw. 53 Prozent die Mehrheit. Überproportional vertreten waren jüdische Erwerbstätige auch in den Bereichen Foto, Drogistik, Leder-, Buch-, Hut- und Fleischhandel, ${ }^{73}$ wobei diese Berufe überproportional oft als Selbstständige ausgeübt wurden. ${ }^{74}$ Ein knappes Viertel der erwerbstätigen Juden und Jüdinnen arbeitete im Bereich Gewerbe und Industrie, hinsichtlich Letzterem vor allem in der Holzund Bekleidungsindustrie. Fest verankert war die jüdische Bevölkerung seit Beginn des Jahrhunderts auch in der Nahrungsmittel- und Textilindustrie. Weiters nahmen Juden und Jüdinnen vor allem im Kürschnergewerbe (68 Prozent), unter den Juwelieren (40 Prozent), als ModistInnen (34 Prozent), UhrmacherInnen (35 Prozent) und ZahntechnikerInnen (31 Prozent) hohe Anteile ein. ${ }^{75}$

70 Maderegger, Ständestaat, 224 ff., 240 ff.; Beller, Wien, 72, 78; vgl. auch Bruce F. Pauley, Political Antisemitism in Interwar Vienna. In: Ivar Oxaal, Michael Pollak, Gerhard Botz (Hg.), Jews, Antisemitism and Culture in Vienna (London 1987) 152-173.

71 Leo Goldhammer, Ueber die Berufsgliederung der Wiener Juden. In: Der Jude. Organ für das arbeitende Palästina 5 (1938), Nr. 2, 14. Jänner 1938, 2-3, hier 2; Goldhammer, Juden Wiens, 54 f., 58. Lichtblau, Integration, 478-483; Hans Witek, „Arisierungen“ in Wien. Aspekte nationalsozialistischer Enteignungspolitik 1938-1940. In: Emmerich Tálos, Ernst Hanisch, Wolfgang Neugebauer, Reinhard Sieder (Hg.), NS-Herrschaft in Österreich. Ein Handbuch (Wien 2000) 795-816, hier 812, FN 3. Vgl. zur Berufsgliederung der Wiener Bevölkerung allgemein Magistratsabteilung für Statistik, Jahrbuch 1930-1935, 16.

72 Maderegger, Ständestaat, 240; vgl. zu Beschäftigten im öffentlichen Dienst auch Bundesamt für Statistik, Handbuch 1937, 215.

73 Goldhammer, Berufsgliederung, 3; Maderegger, Ständestaat, 220.

74 Goldhammer, Juden Wiens, 55-58; vgl. auch Fuchs, 10.

75 Goldhammer, Berufsgliederung, 3; Lichtblau, Partizipation, 246; Gertraud Fuchs, Die Vermögensverkehrsstelle als Arisierungsbehörde jüdischer Betriebe (Dipl.-Arbeit Wirtschaftsuniversität Wien 1989) 11. 
Tab. 2: Anteil jüdischer Erwerbstätiger in ausgewählten Berufsgruppen in Wien in Absolutzahlen und in Prozent für das Jahr 1936 nach Goldhammer. ${ }^{76}$

\begin{tabular}{lcll}
\hline Berufsgruppe & insgesamt & $\begin{array}{l}\text { davon jüdische } \\
\text { Erwerbstätige }\end{array}$ & $\begin{array}{l}\text { Anteil jüdischer } \\
\text { Erwerbstätiger } \\
\text { in Prozent }\end{array}$ \\
\hline Freie Berufe: & & & \\
RechtsanwältInnen & & & \\
ÄrztInnen & 2.163 & 1.341 & 62 \\
ZahnärztInnen & 3.268 & 1.542 & 47,2 \\
Angestellte: & 711 & 446 & 62,7 \\
Bankangestellte & & & \\
Universitätsprofessoren & & & 18,1 \\
\hline & 6.141 & 569 & 28,6 \\
\hline
\end{tabular}

Tab. 3: Berufsgliederung der jüdischen Erwerbstätigen Wiens im Jahr 1934 in Prozent nach Goldhammer, basierend auf der Volkzählung $1934 .^{79}$

\begin{tabular}{|c|c|c|}
\hline Berufssektor & $\begin{array}{l}\text { erwerbstätige jüdische } \\
\text { Bevölkerung in Prozent }\end{array}$ & $\begin{array}{l}\text { sonstige erwerbstätige } \\
\text { Bevölkerung in Prozent }\end{array}$ \\
\hline Landwirtschaft & 0,7 & 1,1 \\
\hline Gewerbe und Industrie & 23,4 & 48,5 \\
\hline Handel und Verkehr & 54,5 & 25,1 \\
\hline Öffentliche Dienste und freie Berufe & 20,1 & 11,1 \\
\hline Ohne Angabe & - & 3,5 \\
\hline Häusliche Dienste & 1,3 & 10,7 \\
\hline Gesamt $^{80}$ & 100,0 & 100,0 \\
\hline
\end{tabular}

76 Goldhammer, Berufsgliederung, 3. Vgl. auch Bundesamt für Statistik, Handbuch 1937, 216.

77 Vgl. zu jüdischen Rechtsanwältinnen Elisabeth Berger, „Scharfes Denken, also juristisches Denken ist weniger Sache der Frau“. In: Österreichisches Anwaltsblatt Heft 3 (2013) 113-121; sowie die Website der Wiener Rechtsanwaltskammer mit entsprechenden Ausführungen: https://www.rakwien.at/?seite=kammer\&bereich=geschichte (Mai 2017).

78 Inklusive Titularprofessoren ohne Ordinariat. Goldhammer, Berufsgliederung, 3. Vgl. auch Maderegger, Ständestaat, 253 mit biographischen Beispielen.

79 Goldhammer, Berufsgliederung, 2; siehe auch Maderegger, Ständestaat, 220; Mejstrik et al., Berufsschädigungen, 98f.; Fuchs, Vermögensverkehrsstelle, 9. Eine Zusammenstellung der wirtschaftlichen Zugehörigkeit mit Vergleichszahlen für Wien und Gesamtösterreich findet sich auch in Bundesamt für Statistik, Volkszählung 1934, Textheft, 156.

80 An Absolutzahlen führt Goldhammer basierend auf der Volkszählung 1934 hierzu neben 853.480 nichtjüdischen Erwerbstätigen 102.760 jüdische Erwerbstätige an. Dieser unrealistisch hohe Wert gründet in der Inklusion von faktisch Erwerbslosen, InhaberInnen von Gewerbe- 
Von den österreichischen Juden und Jüdinnen stand rund ein Drittel im Erwerbsleben (36,5 Prozent, ca. 65.000 Personen, Wert von Anfang 1938); ${ }^{81}$ sie bildeten damit rund 2,3 Prozent aller Erwerbstätigen in Österreich. ${ }^{82}$ Die Erwerbsquote der jüdischen Bevölkerung war im Vergleich zur durchschnittlichen Erwerbsquote in Österreich (50,1 Prozent; Wert von 1934) geringer. Eine Ursache dafür lag wohl in der Überalterung dieser Bevölkerungsgruppe und in der geringeren Erwerbstätigkeit von jüdischen Frauen. ${ }^{83}$ In einer aus 1953 stammenden, retrospektiven Schätzung wurden die jüdischen Erwerbstätigen in einem Ausmaß von 50 Prozent dem ,wohlhabenderen bürgerlichen Mittelstand“ zugerechnet. ${ }^{84}$

\section{Dramatischer Epilog: 1938 bis 1945}

Zum Zeitpunkt des „Anschlusses“ am 12. März 1938 lebten wegen Geburtenrückgangs und einer geringfügigen Auswanderung etwas weniger Juden und Jüdinnen in Österreich als im Jahr 1934; nach den Berechnungen des Historikers Jonny Moser handelte es sich um 167.249 Personen in Wien und 14.633 in den Bundesländern (gesamt: 181.882). ${ }^{85}$ Rund 24.000 weitere Personen erhielten durch die NS-Definition der ab 20. Mai 1938 in Österreich geltenden Nürnberger Gesetze den „Personenstand“ eines Juden oder einer Jüdin zugeschrieben, ungeachtet dessen, ob sie der IKG angehörten, sich mit dem Judentum identifizierten oder religiös lebten. ${ }^{86}$ Für die NS-Behörden galt wegen der zahl-

scheinen und Geringstverdienenden. Goldhammer, Berufsgliederung, 2. Durch diesen Umstand können etwaige Verzerrungen in den Relativwerten nicht ausgeschlossen werden.

81 Clemens Jabloner et al., Schlussbericht der Historikerkommission der Republik Österreich: Vermögensentzug während der NS-Zeit sowie Rückstellungen und Entschädigungen seit 1945 in Österreich. Zusammenfassungen und Einschätzungen (Veröffentlichungen der Österreichischen Historikerkommission 1, Wien/München 2003) 138.

82 Fuchs, Vermögensverkehrsstelle, 6, 8; Mejstrik et al., Berufsschädigungen, $98 \mathrm{f}$.

83 Jabloner, Schlussbericht, 138; Mejstrik et al., Berufsschädigungen, 98 f.; Goldhammer, Juden Wiens, 55.

84 Mejstrik et al., Berufsschädigungen, $103 \mathrm{f}$.

85 Moser, Demographie, 16; vgl. auch Dokumentationsarchiv des österreichischen Widerstandes (Hg.), Widerstand und Verfolgung in Wien, Bd. 3 (Wien $\left.{ }^{2} 1984\right) 201 \mathrm{f} . ;$ Statistisches Landesamt, Jahrbuch 1938, 34; Gerhard Botz, Nationalsozialismus in Wien. Machtübernahme, Herrschaftssicherung, Radikalisierung 1938/39 (überarb. u. erw. Neuaufl. Wien 2008) 626.

$86 \mathrm{Zu}$ Zahlenangaben vgl. auch den Abschnitt „Demographie der österreichischen Juden 1938-1945“ unter http://www.doew.at/erforschen/publikationen/gesamtverzeichnis/holocaust/ demographie-der-juedischen-bevoelkerung-oesterreichs-1938-1945 (April 2017); etwas andere Zahlen bei Botz, Nationalsozialismus (2008), 626. 
reichen Konversionen bzw. Austritte nach 1918 diese auf die Glaubenszugehörigkeit der Großeltern ${ }^{87}$ abstellende „Schaffung des juristischen Begriffs des Rassejuden [...] für die Feststellung der Volkszugehörigkeit der Juden [als] weit verlässlicher" ${ }^{88}$ als die Zuordnung eines Menschen nach aktuellem Religionsbekenntnis.

Demnach galten $\mathrm{zu}$ Beginn des NS-Regimes in Österreich etwa 206.000 Menschen gemäß den Nürnberger Gesetzen als Juden und Jüdinnen, ${ }^{89}$ wenngleich in den ersten beiden Anschlussjahren 1938 und 19396.186 Personen aus der IKG austraten - vermutlich vorrangig EhepartnerInnen, die zuvor für eine Eheschließung zum Judentum konvertiert waren. ${ }^{90} 1.528$ Personen traten im selben Zeitraum in die jüdische Religionsgemeinschaft ein, wohl um Fürsorgeleistungen oder Hilfe bei der Auswanderung durch die IKG erhalten zu können. ${ }^{91}$ (Aufgrund dieser Zahlen wird häufig von einer leicht reduzierten Anzahl von 201.000 Juden und Jüdinnen im angeschlossenen Österreich ausgegangen; die aus der IKG nach dem „Anschluss“ wieder Ausgetretenen galten nach der nationalsozialistischen Rassedefinition nicht als Juden und Jüdin-

87 Gesetz zum Schutze des deutschen Blutes und der deutschen Ehre, dRGBl I 1935, 1146; Erste Verordnung zum Reichsbürgergesetz vom 14. November 1935, dRGBl I 1935, 1333, hier insb. § 2 Abs 2, § 5. Die Nürnberger Gesetze definierten als Juden und Jüdinnen Angehörige der jüdischen Religion (Mitglieder der Israelitischen Kultusgemeinden) sowie Personen mit drei oder mehr Großelternteilen jüdischen Glaubens, selbst wenn diese getauft oder konfessionslos waren; auch Personen mit zwei Großelternteilen jüdischen Glaubens, wenn diese bei Gesetzeserlass oder danach Mitglied der jüdischen Religionsgemeinschaft oder mit einem Juden/einer Jüdin verheiratet waren, galten als jüdisch. Die Bestimmungen wurden in Österreich am 20. Mai 1938 eingeführt. Vgl. dRGBl I 1938, 594 f., weiter: Jonny Moser, Die Katastrophe der Juden in Österreich 1938-1945. In: Wolfgang Plat (Hg.), Voll Leben und voll Tod ist diese Erde. Bilder aus der Geschichte der jüdischen Österreicher (1190-1945) (Wien 1988) 225-260, hier 239; Stuhlpfarrer, Judenfeindschaft, 160; Lichtblau, Integration, 529 f.; ausführlicher: Rainer Faupel, Klaus Eschen, Gesetzliches Unrecht in der Zeit des Nationalsozialismus. Vor 60 Jahren. Erlaß der Nürnberger Gesetze (Baden-Baden 1997).

88 NSDAP - Gau Wien - Organisationsamt gemeinsam mit der Gemeindeverwaltung des Reichsgaues Wien, Statistische Abteilung (Hg.), Wien im Grossdeutschen Reich. Eine statistische Untersuchung über die Lage Wiens nach Wiedereingliederung der Ostmark in das Deutsche Reich (Wien, Februar 1941). Abgedruckt in: Gerhard Botz, Wien vom „Anschluss“ zum Krieg. Nationalsozialistische Machtübernahme und politisch-soziale Umgestaltung am Beispiel der Stadt Wien 1938/39. Mit einem einleitenden Beitrag von Karl R. Stadler (Wien/München 1978) 589-637, hier 605.

89 Moser, Demographie, 16-20.

90 Lichtblau, Integration, 529; Moser, Demographie, 19, 25 f.; vgl. etwa auch Gemeindeverwaltung, Jahrbuch 1938, 40, 200.

91 Lichtblau, Integration, 529; Moser, Demographie, 25 f.; Gemeindeverwaltung, Jahrbuch 1938, 200. 
nen.) Für die jüdische Bevölkerung, die vom NS-Regime am vehementesten verfolgte Personengruppe, bedeuteten der „Anschluss“ und die stufenweise Einführung von reichsdeutschen Gesetzen in Österreich gesetzlich fundierte Einschränkungen in vielen Lebensbereichen, die Flucht und Untertauchen, Deportation und Ermordung zur Folge hatten.

Vor 1938 waren die Emigrations- und Fluchttendenzen jüdischer BürgerInnen nur schwach ausgeprägt gewesen. Selbst nach dem sogenannten Juli-Abkommen $1936^{92}$ war es zu keiner nennenswerten Erhöhung jüdischer Auswanderung gekommen. Nur rund 1.250 Juden und Jüdinnen waren zwischen 1936 und März 1938 emigriert. ${ }^{93}$ Erst nach dem „Anschluss“ setzte aufgrund der Verfolgungsmaschinerie des NS-Regimes eine Massenfluchtbewegung ein, die in den ersten 24 Monaten nach dem März 1938 bis Anfang 1940 kulminierte. ${ }^{94}$

Mitunter unterstützt von Hilfsorganisationen, ${ }^{95}$ gelang etwa zwei Dritteln der österreichischen Juden und Jüdinnen die Flucht ins Ausland, davon erreichten rund 131.000 ein Asylland. Je 30.000 Flüchtlinge gelangten im Zeitraum zwischen „Anschluss“ und Kriegsausbruch 1939 nach Großbritannien und in die USA, zahlreiche Personen flohen nach China/Shanghai, Palästina, Mittel- und Südamerika und Australien. ${ }^{96}$ Weitere Erst-Zielorte der EmigrantInnen bildeten Italien, die Schweiz, Frankreich und etwa die Stadt Harbin im heutigen China. ${ }^{97}$

Die nach nationalsozialistischen Parametern durchgeführte Volkszählung vom 17. Mai 1939 zählte nur noch 92.982 Juden und Jüdinnen in Wien. ${ }^{98}$ In den

92 Das Juli-Abkommen (1936) kennzeichnete den Beginn einer deutschlandfreundlichen Politik Österreichs, u. a. sah es die Amnestie inhaftierter Nationalsozialisten vor.

93 Moser, Demographie, $11 \mathrm{f}$;; Lichtblau, Partizipation, $250 \mathrm{ff}$.

94 Moser, Demographie, 29-56. Vgl. allgemein etwa auch Helene Maimann, Vergangenheit, die nicht vergeht. NS-Herrschaft in Österreich, 1938-1945. In: Günter Düriegl (Hg.), Das neue Österreich. Die Ausstellung zum Staatsvertragsjubiläum 1955-2005. Oberes Belvedere, 16. Mai bis 1. November 2005 (Wien 2005), oder Florian Freund, Hans Safrian, Die Verfolgung der österreichischen Juden 1938-1945. In: Emmerich Tálos et al. (Hg.), NS-Herrschaft in Österreich. Ein Handbuch (Wien 2000) 767-794.

95 Vgl. etwa Theodor Venus, Alexandra-Eileen Wenck, Die Entziehung jüdischen Vermögens im Rahmen der Aktion Gildemeester. Eine empirische Studie über Organisation, Form und Wandel von „Arisierung“ und jüdischer Auswanderung in Österreich 1938-1941 (Veröffentlichungen der Österreichischen Historikerkommission 20/2, Wien/München 2004).

96 Pulzer, Nachwort, 305; Lichtblau, Integration, 526. Bis 30. November 1939 hatten $126.445 \mathrm{Ju}$ den und Jüdinnen Österreich verlassen können. Stuhlpfarrer, Judenfeindschaft, 170. Siehe auch Moser, Demographie, 28.

97 Moser, Demographie, 58-64.

98 Lichtblau, Integration, 530; Moser, Demographie, 31. 
Bundesländern bzw. Gauen befanden sich zu diesem Zeitpunkt nur noch rund 3.000 jüdische Personen. ${ }^{99}$ Insgesamt lebten zum Zeitpunkt der Volkszählung 1939 noch 96.042 Juden und Jüdinnen im Sinne der Nürnberger Gesetze in Österreich. ${ }^{100}$

Die Auswanderung war für jüngere Personengruppen, die noch in den Arbeitsmarkt der aufnehmenden Staaten integriert werden konnten, zunächst leichter als für ältere und finanzschwache Juden und Jüdinnen. ${ }^{101}$ Mit der Verschärfung der Einreisebestimmungen nach England und Lateinamerika, dem Kriegsbeginn sowie dem Beginn der Deportationen (d. h. der staatlich angeordneten, zwangsweisen Verschickung der jüdischen Restbevölkerung in Ghettos oder Konzentrationslager) und Maßnahmen im Zusammenhang mit der geplanten „Endlösung“ gingen die Möglichkeiten einer Emigration für die Ende 1940 noch 61.135 in Wien befindlichen Juden und Jüdinnen schließlich stark zurück. ${ }^{102}$ Per August 1941 wurde die Ausreise arbeitsfähiger Juden und Jüdinnen nicht mehr gestattet; und ab Ende Oktober 1941 jede Emigration verboten. ${ }^{103}$ Insgesamt wurden etwa 48.000 Menschen insbesondere ab Februar 1941 aus Wien deportiert; von ihnen überlebten nur rund 2.000 die Zeit des NS-Regimes. ${ }^{104}$ Ende 1942 befanden sich nach Moser nur noch 8.052 Juden und Jüdinnen in Wien, zu Kriegsende 5.512. Sie lebten zum überwiegenden Teil in „Mischehe“ oder waren entweder Angestellte der IKG, des jüdischen Krankenhauses, dort untergebrachte PatientInnen oder Ausländer. ${ }^{105}$ Nur rund

99 2.013 Personen in Niederdonau, 228 in Oberdonau, 609 in der Steiermark, in Kärnten 54, in Salzburg 47, in Tirol 85 und in Vorarlberg 24. Die meisten Juden und Jüdinnen in den Bundesländern waren nach dem „Anschluss“ bereits nach Wien gezogen oder emigriert. Moser, Demographie, 31.

100 Moser, Demographie, 31. Leicht abweichende Zahlen für die Wiener Juden und Jüdinnen laut Volkszählung 1939 (91.480 Juden) bei NSDAP, Wien, 607; siehe auch Stuhlpfarrer, Judenfeindschaft, 170; Lichtblau, Integration, 528, $534 \mathrm{f}$.

101 Gerhard Botz, Stufen der Ausgliederung der Juden aus der Gesellschaft. Die österreichischen Juden vom „Anschluss“ zum „Holocaust“. In: Zeitgeschichte 14 (1986) 372; Stuhlpfarrer, Judenfeindschaft, 171.

102 Moser, Demographie, 29-56; Botz, Stufen, 372.

103 Moser, Demografie, 46.

104 Stuhlpfarrer, Judenfeindschaft, 175 f.; Botz, Stufen, 359-378, 373 f. Vgl. den Abschnitt „Demographie der österreichischen Juden 1938-1945“ unter http://www.doew.at/erforschen/ publikationen/gesamtverzeichnis/holocaust/demographie-der-juedischen-bevoelkerungoesterreichs-1938-1945 (April 2017); Moser, Demographie, 49, 80 ff.

105 Moser, Demographie, 47, 52 ff.; Lichtblau, Integration, 533. Vgl. auch die NS-Statistik dazu in NSDAP, Wien, 606. 
1.000 Menschen überlebten in Wien als „U-Boote“. ${ }^{106}$ Von jenen Personen, die zunächst in ein anderes europäisches Land fliehen hatten können, fielen weitere 16.000 dort der nationalsozialistischen Verfolgung zum Opfer. ${ }^{107}$ Insgesamt wird von rund 65.000 im Holocaust ermordeten jüdischen ÖsterreicherInnen ausgegangen. ${ }^{108}$

Nur ein geringer Teil der in die Flucht getriebenen Personen kehrte zu einem späteren Zeitpunkt wieder nach Österreich zurück. Von den vertriebenen 131.000 Juden und Jüdinnen remigrierten bis $\mathrm{zu}$ den 1960er-Jahren nur rund 9.000 Personen - also etwa sieben Prozent. ${ }^{109}$ Die Fluchtwege und weiteren Lebensverläufe, insbesondere von SportlerInnen und SportfunktionärInnen, und auch der mit der Emigration einhergegangene Kulturtransfer sind bislang nur vereinzelt wissenschaftlich bearbeitet worden; weitere Forschungsprojekte $\mathrm{zu}$ diesem in der historischen Arbeit zuweilen schwer erschließbaren Thema sind aber im Gange. ${ }^{110}$

106 Dieter J. Hecht, Eleonore Lappin-Eppel, Michaela Raggam-Blesch, Topographie der Shoah. Gedächtnisorte des zerstörten jüdischen Wien (Wien 2015) 528.

107 Vgl. den Abschnitt „Demographie der österreichischen Juden 1938-1945“ unter http:// www.doew.at/erforschen/publikationen/gesamtverzeichnis/holocaust/demographie-der-juedi schen-bevoelkerung-oesterreichs-1938-1945 (April 2017).

108 Botz, Stufen, 374.

109 Lichtblau, Integration, 537. Pulzer spricht von insgesamt maximal 20 Prozent RemigrantInnen nach 1945. Pulzer, Nachwort, 309. Vgl. auch Bericht des Präsidiums der Israelitischen Kultusgemeinde Wien über die Tätigkeit in den Jahren 1945 bis 1948 (Wien 1948) 48 ff., sowie Brigitte Bailer, Überlebende des Holocaust in der Zweiten Republik - eine Skizze. In: Dokumentationsarchiv des österreichischen Widerstandes (Hg.), Feindbilder (Wien 2015) 113-139; Wolfgang Neugebauer, Siegwald Ganglmair, Remigration. In: Dokumentationsarchiv des österreichischen Widerstandes, Jahrbuch 2003 (Wien 2003) 96-102, hier 98.

110 Kürzlich erschienen: David Bolchover, The Greatest Comeback. From Genocide To Football Glory, the Story of Béla Guttman (London 2017); Vida Bakondy, Montagen der Vergangenheit. Flucht, Exil und Holocaust in den Fotoalben der Wiener Hakoah-Schwimmerin Fritzi Löwy (1910-1994) (Göttingen 2017); Alexander Juraske, „Judenxandl und Stadtpelz“. Die vergessenen jüdischen Funktionäre des First Vienna Football Club 1894. In: Aschkenas 27, H. 1 (2017) 39-56, hier 52-54; Johann Skocek, Mister Austria. Das Leben des Klubsekretärs Norbert Lopper. Fußballer, KZ-Häftling, Weltbürger (Wien 2014). Vgl. weiter Karen Propp, The Danube Maidens. Hakoah Vienna Girls' Swim Team in the 1920s and 1930s; David Forster, Georg Spitaler, Die Fußballmeister. Lebenswege der Hakoah-Spieler der Zwischenkriegszeit; Susanne Helene Betz, Von der Platzeröffnung bis zum Platzverlust. Die Geschichte der Hakoah Wien und ihrer Sportanlage in der Krieau. Alle in: Betz, Löscher, Schölnberger (Hg.), „... mehr als ein Sportverein“. 


\section{Wiener Sport in der Zwischenkriegszeit in Zahlen}

Die Wiener Sportlandschaft gestaltete sich in den 1920er- und 1930er-Jahren sehr heterogen und vielfältig. Sportvereine divergierten nach sportlicher Disziplin, religiöser und politischer Gesinnung, Berufsstand, sozialer Schicht und Zugehörigkeit, Alter, Geschlecht und Wohnbezirk. Die Zahl der jüdischen Turnund Sportvereine in Wien belief sich in den 1920er-Jahren auf mehrere Dutzend Klubs; nach der Weltwirtschaftskrise bis 1938 bestanden noch rund 20 Vereine, darunter die Hakoahklubs und die Makkabi-Turnvereine. ${ }^{111}$ Die jüdischen Vereine brachten in den 1920er- und 1930er-Jahren äußerst erfolgreiche SportlerInnen hervor. ${ }^{112}$

Für das Wien des Jahres 1929 dokumentiert das Statistische Jahrbuch der Stadt ${ }^{113}$ zunächst insgesamt 1.225 verschiedene Sportvereine, die in 47 Verbänden organisiert waren und insgesamt 106.808 Mitglieder zählten (siehe Tabelle 4). Die mitgliederstärkste bzw. beliebteste Sportart bildete der Fußball mit beinahe 31.000 Spielern, auf ihn folgten die Disziplinen Schwimmen, Eislaufen und Skifahren. Frauen stellten durchschnittlich knapp zwei Fünftel (39 Prozent) der Mitglieder; mit höheren Anteilen waren Frauen im Schwimmsport

111 Paul Földes, Die Arbeiten des Jüdischen Turn- und Sportverbandes Österreichs. In: Jüdischer Sport. Sport, Turnen, Touristik. Offizielles Organ des Makkabi-Weltverbandes und des jüdischen Sport- und Turnverbandes Österreich in Wien Nr. 1 (1925) 2, nennt für 1925 eine Anzahl von 30 jüdischen Sport-und Turnvereinen in ganz Österreich; Jakob Krausz, Michael Winkler (Hg.), Jüdisches Leben in Österreich in Wort und Bild, mit Gemeinde- und Vereinskalender für das Jahr 5686 (Wien 1925-1926) 74, nennen 20 jüdische Vereine in Wien; vgl. weiter Arthur Baar (Hg.), 50 Jahre Hakoah 1909-1959 (Tel Aviv 1959) 54; Nachrichtenblatt des Schwimmklubs „Hakoah“ Nr. 326 (15. 5. 1937) 1; ÖStA, AdR, ZNsZ Stiko Wien, Mappe 31-N 14, Mitgliederstatistik des Jüdischen Turn- und Sportverbandes Österreichs im „Makkabi-Weltverband“ vom 30. 6. 1936; Duizend-Jensen, Gemeinden, 48 f.; Ignaz Hermann Körner, Lexikon jüdischer Sportler in Wien. 1900-1938. Hg. u. ed. von Marcus G. Patka im Auftrag des Jüdischen Museums Wien (Wien 2008) 206f.; Matthias Marschik, Von jüdischen Vereinen und „Judenclubs“. Organisiertes Sportleben um die Jahrhundertwende. In: Adunka, Lamprecht, Traska (Hg.), Jüdisches Vereinswesen, 225-244, hier 235; siehe auch Kapitel 4 und 10.

112 Einschließlich der Olympischen Spiele 1932 errangen jüdische SportlerInnen z.B. drei Gold-, sechs Silber- und zehn Bronzemedaillen für Österreich. Vgl. Lichtblau, Integration, 506. Etwas andere (teilweise unrichtige) Zahlen bei Hans Morgenstern, Über den Anteil von Juden am Sport in Österreich. In: Klaus Lohrmann (Hg.), 1000 Jahre österreichisches Judentum (Ausstellungskatalog, Eisenstadt 1982) 193-199, hier 195 ff. Pierre Gildesgame Maccabi Sports Museum, Ramat Gan, Israel, Maccabi Austria Files, 4-01-050 [PDF-Dokument]: Ignaz Körner, Die Juden Wiens bei den Olympiaden. Manuskript, 76-78, enthält ebenfalls eine Aufstellung.

113 Magistratsabteilung für Statistik, Jahrbuch 1929 (NF, 2. Jahrgang), 281-285. 
(47 Prozent) und beim Eislaufen (49 Prozent) vertreten. ${ }^{114}$ Rein männliche Sportgattungen bildeten Eishockey, Boxen und Schießen. ${ }^{115}$ Weiters existierten 129 in fünf Verbänden (Christlich-deutsche Turnerschaft Gau Wien, Deutscher Turnerbund Gau Wien, Dolnorakouska župa sokolska [Niederösterreichischer Sokolgau], Wiener Arbeiter-Turnverein, Deutsch-österreichischer Turnverein) $)^{116}$ organisierte Turngruppen und Turnvereine, die beinahe 49.000 Mitglieder zählten; hier stellten Frauen in etwa die Hälfte der SportlerInnen. ${ }^{117}$

Für die Wiener Vereine bot die Stadt einiges an Infrastruktur, wenngleich in der Zwischenkriegszeit das Angebot an Sportstätten vielfach bemängelt wurde. ${ }^{118}$ Ende der 1920er-Jahre verwaltete die Gemeinde Wien allein 78 AußenSportflächen (26 Fußballplätze, ein Golfplatz, zwölf Eislauf- und Tennisplätze, 29 Spielplätze, neun Turnplätze, ein Schießplatz) und sechs geschlossene Sportstätten (drei Turnsäle und drei Schwimmhallen). ${ }^{119}$

Die Wiener Bergsteigervereine und Sektionen waren Ende der 1920er-Jahre mit insgesamt 133.043 Mitgliedern (davon rund 100.000 Vollzahlende, die weiteren Familienangehörige) noch stärker nachgefragt als die klassischen Sportund Turnklubs. Die zu dieser Gruppe zählenden 221 Vereine (Sektionen) waren in fünf Verbänden - Naturfreunde, Österreichische Bergsteigervereinigung, Österreichischer Gebirgsverein, Österreichischer Touristenklub und Deutschösterreichischer Alpenverein - organisiert und unterhielten 152 Berghütten; die meisten dieser Herbergen (59) betreute der Deutschösterreichische Alpenverein. ${ }^{120}$

114 Magistratsabteilung für Statistik, Jahrbuch 1929 (NF, 2. Jahrgang), 282. Schwimmen: 24.908 Mitglieder, davon 11.625 Frauen (Zahlen von 1928); Eislaufen: 12.963 Mitglieder, davon 6.382 Frauen (Zahlen von 1929).

115 Die Statistik von 1929 enthält nicht zu allen Sportarten nach Geschlechtern differenzierende Mitgliederdaten. Magistratsabteilung für Statistik, Jahrbuch 1929 (NF, 2. Jahrgang), 282-285. 116 Der Jüdische Turn- und Sportverband Österreichs im „Makkabi-Weltverband“ war aus unbekannten Gründen in der amtlichen Statistik nicht aufgezählt. Siehe zu diesem Verband Kapitel 4, 8 und 10.

117 Magistratsabteilung für Statistik, Jahrbuch 1929 (NF, 2. Jahrgang), $285 \mathrm{f}$.

118 Vgl. etwa die Arbeiter-Zeitung (31. 5. 1928) 4; Österreichisches Sportwesen. In: Reichspost (22. 2. 1920) 12; weiters: Das Kleine Blatt (29. 1. 1931) 14.

119 Magistratsabteilung für Statistik, Jahrbuch 1929 (NF, 2. Jahrgang), 281. Angabe der Turnhallen ohne die mehreren Hundert schulischen Turnsäle; im Jahr 1924 etwa gab es davon in Wien 416. Bundesamt für Statistik, Handbuch 1925, 162.

120 Magistratsabteilung für Statistik, Jahrbuch 1929 (NF, 2. Jahrgang), 283. Mit „Deutschösterreichischem Alpenverein“ meint das Jahrbuch offenbar den Deutschen und Oesterreichischen Alpenverein (D.u.Oe.A.V.). 
Tab. 4: Übersicht über Sport-, Turn-, und Alpinvereine in Wien im Jahr 1929. ${ }^{121}$

\begin{tabular}{|c|c|c|c|c|c|c|}
\hline Vereine & $\begin{array}{l}\text { Anzahl- } \\
\text { Verbände }\end{array}$ & $\begin{array}{l}\text { Anzahl- } \\
\text { Vereine } \\
\text { (Sektio- } \\
\text { nen) }\end{array}$ & $\begin{array}{l}\text { Anzahl ver- } \\
\text { wendeter } \\
\text { Sportplätze }\end{array}$ & Mitglieder & $\begin{array}{l}\text { davon } \\
\text { Männer } \\
\text { inkl. Nach- } \\
\text { wuchs }\end{array}$ & $\begin{array}{l}\text { davon } \\
\text { Frauen } \\
\text { inkl. Nach- } \\
\text { wuchs }\end{array}$ \\
\hline Sportvereine & 47 & 1.225 & & 106.808 & & \\
\hline Golf & 1 & 1 & 1 & 387 & 230 & 157 \\
\hline Tennis & 2 & 23 & 22 & 563 & - & - \\
\hline Hockey & 1 & 17 & 7 & 700 & - & - \\
\hline Leichtathletik & 4 & 63 & 41 & 8.946 & - & - \\
\hline Handball & 4 & 67 & 48 & 3.665 & 2.486 & 1.179 \\
\hline Fußball & 2 & 532 & 81 & 30.795 & - & - \\
\hline Eislaufen & 4 & 23 & 9 & 12.963 & 6.581 & 6.382 \\
\hline Boxen & 1 & 7 & 7 & 600 & 600 & 0 \\
\hline Kraftsport & 2 & 112 & 5 & 3.011 & 2.933 & 78 \\
\hline Eishockey & 2 & 23 & 11 & 638 & 638 & - \\
\hline $\begin{array}{l}\text { Schlitten- } \\
\text { sport }\end{array}$ & 1 & 1 & 1 & 71 & 42 & 29 \\
\hline Skisport & 6 & 76 & - & 11.034 & - & - \\
\hline Schwimmen & 5 & 102 & 66 & 21.024 & - & - \\
\hline Rudern & 4 & 16 & - & 1.156 & - & - \\
\hline Kajak & 2 & 10 & - & 715 & - & - \\
\hline Radfahren & 2 & 67 & 1 & 3.706 & - & - \\
\hline Reiten & 1 & 1 & 1 & 77 & 69 & 8 \\
\hline Schießen & 3 & 70 & 67 & 6.154 & 6.154 & - \\
\hline Fechten & - & 14 & 14 & 603 & 538 & 65 \\
\hline Turnvereine & 5 & 129 & $\begin{array}{l}307 \text { (davon } \\
261 \text { Hallen) }\end{array}$ & $48.757^{122}$ & 24.208 & 24.549 \\
\hline $\begin{array}{l}\text { Bergsteiger- } \\
\text { vereine (samt } \\
\text { Sektionen) }\end{array}$ & 5 & 221 & $\begin{array}{l}152 \text { (Berg- } \\
\text { hütten) }\end{array}$ & $\begin{array}{l}133.043 \\
(100.220 \\
\text { Vollzah- } \\
\text { lende) }\end{array}$ & - & - \\
\hline
\end{tabular}

-: keine Angabe

Nach den politischen Veränderungen in Österreich 1933/34 und dem Verbot sozialdemokratischer Vereine sowie der (vorübergehenden) Auflösung deutschnationaler Einrichtungen ${ }^{123}$ stellte sich die Sportlandschaft für das Jahr 1935 deut-

121 Magistratsabteilung für Statistik, Jahrbuch 1929 (NF, 2. Jahrgang), 281-285. Wenn Angaben zu 1929 fehlten, wurde auf die Zahlen der Jahre 1927 und 1928 zurückgegriffen. Ebenda, 281 f., sowie: Magistratsabteilung für Statistik, Jahrbuch 1929 (NF, 1. Jahrgang), 256-259. Bemerkenswert ist, dass die Angaben zur Anzahl von Wiener Vereinen und Verbänden stark schwanken.

122 Magistratsabteilung für Statistik, Jahrbuch 1929 (NF, 2. Jahrgang), 284: 48.271, Grund der Differenz unklar.

123 Die Vereine des Deutschen Turnerbunds wurden im August 1934 zunächst verboten, konnten sich unter bestimmten Bedingungen (Überwachung) aber reaktivieren. Vgl. Matthias 
lich reduziert dar. Die Anzahl der Sportvereine war mit insgesamt $813 \mathrm{im}$ Vergleich zu 1929 um ein Drittel geringer. ${ }^{124}$ Die bestehenden Vereine profitierten von der Dezimierung der Vereinslandschaft durch die Neuzuteilung ehemaliger Plätze aufgelöster Klubs. ${ }^{125}$ Nichtsdestotrotz zeigte sich die sportliche Vereinslandschaft aber weiterhin sehr vielfältig. Das Statistische Jahrbuch der Stadt Wien von 1935 listet allein 31 verschiedene Sportverbände auf, denen je nach Sportart jeweils eine unterschiedliche Anzahl der 813 ausgewiesenen Wiener Sportvereine angehörte. So waren etwa 284 Klubs Mitglied beim Wiener Fußballverband (WFV), dem Eishockeyverband gehörten 22 Vereine und dem Handballbund 45 Klubs an. Insgesamt weist die Statistik für 1935 rund 62.854 Vereinsmitglieder aus - also nur noch 59 Prozent des Wertes von 1929 -, wobei Frauen zwischen 15 bis 20 Prozent stellten. Frauen waren wie schon 1929 nicht in jeder Sportart vertreten bzw. nahmen nicht in allen aufgelisteten Disziplinen an Wettkämpfen teil. Sie scheinen etwa beim Boxen, Trab- und Polosport, Tischtennis, in Schützenvereinen oder Eishockey in der Statistik des Jahres 1935 nicht auf. ${ }^{126}$ Darüber hinaus sind im Jahr 1935 weiterhin fünf Turnverbände bzw. Vereinigungen - die Christlich-deutsche Turnerschaft, der (reduzierte) Deutsche Turnerbund, der Allgemeine Turn- und Sportverein ${ }^{127}$, die Gruppe der fünf Makkabi-Turnvereine und der Verband der Sokol-Turnvereine - dokumentiert, denen nach dem Verbot der Arbeiterturnvereine bzw. den Auflösungen von Vereinen des Deutschen Turnerbunds nunmehr 108 Turnvereine angehörten. Insgesamt zählten diese Turnklubs mit 16.911 Mitgliedern um zwei Drittel weniger SportlerInnen als 1929. Die Hälfte von ihnen waren Frauen bzw. Mädchen. Die fünf Makkabi-Turnvereine lagen nach der mitgliederstärksten Christlich-deutschen Turnerschaft mit ihren insgesamt 1.030 Mitgliedern an vierter Stelle. ${ }^{128}$

Das Geschlechterverhältnis in den jüdischen Sportvereinen glich Mitte der 1930er-Jahre im Übrigen (soweit Aussagen auf Basis des nur rudimentär vor-

Marschik, Turnen und Sport im Austrofaschismus (1934-1938). In: Emmerich Tálos, Wolfgang Neugebauer (Hg.), Austrofaschismus. Politik - Ökonomie - Kultur 1933-1938 (Münster/London/Wien 2005) 372-389, hier 381, sowie Roland Schmidl, Der deutsche Turnerbund (1919) und seine Relevanz in der Ersten Republik Österreich (Diss. Universität Wien 1978) $234 \mathrm{ff}$.

124 Magistratsabteilung für Statistik, Jahrbuch 1930-1935, 197.

125 Marschik, Austrofaschismus, 379.

126 Magistratsabteilung für Statistik, Jahrbuch 1930-1935, 197. Da die Zahlen zu einigen Verbänden nicht zwischen den Geschlechtern differenzieren, können zum Frauenanteil nur Schätzwerte angegeben werden.

127 Hierbei handelte es sich um die Fortführung des Deutsch-österreichischen Turnvereins. 128 Magistratsabteilung für Statistik, Jahrbuch 1930-1935, 201. Dort auch die Zahlenangaben für 1930-1934; 1934 erreichte die Mitgliederzahl in Turnvereinen aufgrund der politischen Interventionen mit 11.700 ihren Tiefststand. 
handenen Zahlenmaterials möglich sind) jenem der sonstigen Wiener Sportlandschaft. Frauen stellten zwar nie deutlich mehr als die Hälfte der Mitglieder der Klubs, ihre Anteile lagen aber meist im zweistelligen Bereich. Unter den Mitgliedern der Makkabi-Turnvereine befanden sich 1935542 (53 Prozent) Sportlerinnen; ${ }^{129}$ von den insgesamt 1.568 Mitgliedern aller Wiener Hakoahvereine bildeten Frauen im Jahr 1936 rund ein Drittel (509; 32 Prozent); im Hakoah-Schwimmklub stellten sie beinahe die Hälfte der 605 Aktiven (272; 45 Prozent). ${ }^{130}$

Hinsichtlich der Bergsteigervereine ist festzuhalten, dass im Jahr 1930 noch 232 solcher Klubs bzw. Sektionen mit insgesamt 102.120 vollzahlenden Mitgliedern gezählt wurden; im Jahr 1935, ohne die sozialdemokratischen Alpinklubs bzw. Verbände wie jener der Naturfreunde, war die Mitgliederzahl halbiert. In 108 Vereinen (53 beim Deutschösterreichischen Alpenverein und 55 bei der Bergsteigvereinigung) sind insgesamt 43.260 Mitglieder dokumentiert. ${ }^{131}$

Nachdem bis Ende 1937 die Anzahl der Wiener Sportklubs wieder angestiegen war und bei 849 Sportvereinen, 114 in sechs Verbänden organisierten Turnvereinen und 118 Alpinvereinen bzw. Sektionen in drei Vereinigungen (inkl. Bergfreunde $)^{132}$ gelegen hatte, ${ }^{133}$ verringerte sich die Diversität der Vereinslandschaft nach dem „Anschluss“ im folgenden Jahr massiv: Ende 1938 dokumentiert die Statistik der Stadt Wien nur noch 650 dem NS-Reichsbund für Leibesübungen, Kreis Wien, zugehörende Wiener Sportvereine - die Hälfte (53 Prozent) des Werts von 1929 -, 93 Turnvereine, die in zwei Verbänden organisiert waren (72 Prozent des Werts von 1929), und 96 Bergsteigervereine (43 Prozent des Werts von 1929). ${ }^{134}$ Diesen Veränderungen in der Vereinslandschaft ab dem März 1938 widmet sich Kapitel 10.

129 Inklusive Mädchen und Frauen unter 18 Jahren. Magistratsabteilung für Statistik, Jahrbuch 1930-1935, 201.

130 ÖStA, AdR, ZNsZ Stiko Wien, Mappe 31-N 14, Mitgliederstatistik des Jüdischen Turn- und Sportverbandes Österreichs im „Makkabi-Weltverband“ vom 30. 6. 1936; vgl. auch Kapitel 4; zur Hakoah siehe auch Kapitel 10.

131 Magistratsabteilung für Statistik, Jahrbuch 1930-1935, 202.

132 Die Bergfreunde bildeten einen Ersatzverein für die 1934 aufgelösten Naturfreunde. Vgl. Österr. Sport- und Turnfront (Hg.), Sportjahrbuch 1935. 4. Jahrgang des Körpersport-Jahrbuches des Österreichischen Hauptverbandes für Körpersport (Wien 1935) 332-335.

133 Magistratsabteilung für Statistik (Hg.), Statistisches Jahrbuch der Stadt Wien 1937 (NF, 4. Band, Wien o. J.) 220, 226. Die Angaben zu den Turnvereinen enthalten keine Makkabi-Vereine. 134 Gemeindeverwaltung des Reichsgaues Wien (Hg.), Statistisches Jahrbuch der Stadt Wien 1938 (NF 5, Wien o. J.) 193, 198. Die Mitgliederzahlen bei den Sportvereinen erhöhten sich, wohl im Zuge der ideologischen Forcierung des Sports durch das NS-Regime bis Ende 1938 auf 124.891 (Frauenanteil rund 29 Prozent). Bei den Turnvereinen sanken die Mitgliederzahlen auf 12.000 (samt Mädchen; Frauenanteil: 53 Prozent), bei den Alpenvereinen auf 30.781 (Vollzahlende). 